\title{
Health system challenges: An obstacle to the success of isoniazid preventive therapy
}

\author{
E I Okoli, ${ }^{1}$ MBBS, PG Dip HIV/AIDS Management, MPhil (HIV/AIDS Management), MPH; L Roets, ${ }^{2}$ PhD \\ ${ }^{1}$ Department of Internal Medicine, Dr George Mukhari Academic Hospital, Garankuwa, Pretoria, South Africa \\ ${ }^{2}$ Department of Health Studies, College of Human Sciences, University of South Africa, Pretoria
}

Corresponding author: E I Okoli (docokoli@yahoo.com)

\begin{abstract}
Background. The researchers identified infection with HIV as the strongest risk factor in the reactivation of latent tuberculosis (TB) infection or progression to active disease. Isoniazid preventive therapy (IPT) is one of the interventions recommended by the World Health Organization and the South African (SA) National Department of Health to prevent progression to active TB disease in people living with HIV. Adherence to IPT is therefore the responsibility of healthcare clients and clinicians.

Objectives. To describe the incidence of TB among clients who received IPT, rates of completing and not completing IPT among those who started it, and the reasons for non-completion.

Methods. A quantitative, non-experimental, descriptive retrospective cohort study was undertaken. The clinic records of 104 HIV-positive adults receiving care at a clinic in SA who started IPT between 1 July 2010 and 30 November 2011 were analysed.

Results. Sixty-six of 104 study respondents (63.5\%) completed the IPT course. None of the respondents who completed IPT was diagnosed with $\mathrm{TB}$, and $86.8 \%$ of the respondents who did not complete the programme did so because of the poor quality of healthcare they received, and not by their own choice.

Conclusion. The study results strengthened the findings of similar local and international studies that IPT is advantageous in the prevention of TB. The finding that so many patients did not complete the programme as a result of drug dispensing or prescription problems is alarming, and revealed a major shortcoming in the healthcare system.
\end{abstract}

S Afr Med J 2016;106(11):1079-1081. DOI:10.7196/SAMJ.2016.v106i11.10741

With an estimated 5.6 million people living with HIV in 2011, South Africa (SA)'s epidemic remains the largest in the world. ${ }^{[1]} \mathrm{SA}$ is also the country with the third-highest tuberculosis (TB) burden globally, and is only lagging behind countries such as China and India that have significantly larger populations. ${ }^{[2]}$

TB is also the most common cause of morbidity and mortality in the HIV-infected population in SA, and studies have shown that TB accelerates the progression of HIV/AIDS. ${ }^{[3]}$

Since 1993, when the World Health Organization (WHO) declared TB a global public health emergency because of $7-8$ million recorded cases, the spread of TB increased to about 9 million new cases in $2011 .^{[4]} \mathrm{TB}$ is the second leading cause of death from an infectious disease worldwide after HIV, and accounted for an estimated 1.4 million deaths in 2011. ${ }^{[4]}$ This includes an estimated 430000 deaths from TB among people living with HIV (PLHIV). ${ }^{[4]}$

HIV is the strongest risk factor recognised so far in reactivating latent Mycobacterium tuberculosis infection to active TB disease, or increasing susceptibility to new infection. ${ }^{[5]}$ The risk of active TB increases soon after HIV seroconversion and doubles by the end of the first year of HIV infection. ${ }^{[6]}$

Isoniazid preventive therapy (IPT) is one of the interventions that the World Health Organization (WHO) and the SA National Department of Health (NDoH) recommend to prevent progression to active TB disease in PLHIV. The WHO, with a high quality of evidence, strongly recommends that these individuals receive IPT irrespective of their degree of immunosuppression.

The protective effect of TB preventive therapy is expected to last for approximately 18 months, so the therapy should be given once during this period. ${ }^{[4]}$ The lifetime risk of an infected but immunocompetent individual developing active TB disease is around $10 \%$, with the highest risk in the first 2 years following infection. ${ }^{[7]}$ The risk increases to around $10 \%$ per year in an individual living with HIV. ${ }^{[8]}$

In addition to high rates of reactivation TB, HIV-infected individuals have increased susceptibility to new exogenous infection and rapidly progressive primary disease. HIV-positive individuals who are co-infected with TB are about $21-24$ times more likely to develop active TB disease than those who are HIV-negative. ${ }^{[5]}$

From a public health perspective, IPT is cost-effective, extends life expectancy, reduces the incidence of $\mathrm{TB}$, and promotes savings in medical and social costs for adults with HIV, especially those who are tuberculin skin test (TST)-positive. ${ }^{[5]}$ While individuals who are eligible for antiretroviral therapy (ART) should start it, all PLHIV in whom active TB has been excluded should be started on IPT, with the exception of those who abuse alcohol or have an increased likelihood of side-effects, in which case the $\mathrm{NDoH}$ states that counselling should precede IPT.

Notwithstanding the absence of definitive evidence from randomised controlled trials on the optimal duration of IPT in HIV-infected patients, data from several observational studies demonstrate that IPT is cost-effective and beneficial because it combats low bacillary load latent $\mathrm{TB}$, which serves as a reservoir for recurrent disease.

\section{Methods}

A quantitative non-experimental descriptive retrospective cohort study was performed in a clinic in an industrial area adjacent to a 
formal urban settlement in the iLembe Health District, KwaZuluNatal Province (KZN), SA. The clinic provides the full complement of HIV/AIDS/TB management to the inhabitants of the area.

Using a consecutive sampling method, the researchers collected data between July 2010 and November 2011. They used the daily clinic attendance register to obtain 441 applicable records for the study, and 104 HIV-infected adult clients met the eligibility criteria and formed the sample. The criteria for inclusion were evidence that the patient tested HIV-positive, was $>18$ years of age and had commenced IPT. The cut-off enrolment month of November 2011 applied because the researchers followed the records of the patients for 15 - 30 months to ascertain whether they developed active TB disease after using IPT. They screened the files of all the subjects for TB prior to commencing intake of IPT. Twelve subjects also had their sputum tested for acid-fast bacilli before starting ART. The sputum testing was done because they answered in the affirmative to at least one of the questions in the screening tool and were diagnosed with TB prior to IPT treatment. The researchers also developed a data-capturing instrument based on the objectives of the study and pretested it to gather the required data.

\section{Ethical considerations}

Ethical approval was obtained from the research ethics committee of the University of South Africa (ref. no. HSHDC/92/2012), the Department of Health (KZN) (ref. no. HRKM 011/13) and the iLembe Health District (ref. no. DM2012/09/2289). In order to maintain anonymity and confidentiality, the files were coded but names and file numbers were not entered.

\section{Statistical analysis}

Data were analysed using the SPSS statistical software program, version 21 (IBM, USA). Descriptive statistics were used to describe the demographic characteristics, and analytical statistics using the $\chi^{2}$ test to measure the associations between gender and age distributions of the subjects and those who completed IPT at 6 and 9 months. The $95 \%$ level of confidence ( $95 \%$ confidence interval) and a probability of $p<0.05$ were used as the definition of significance while comparing the groups.

\section{Results}

One hundred and four clinic records of HIV-positive adults receiving care at a clinic and meeting the inclusion criteria were abstracted for

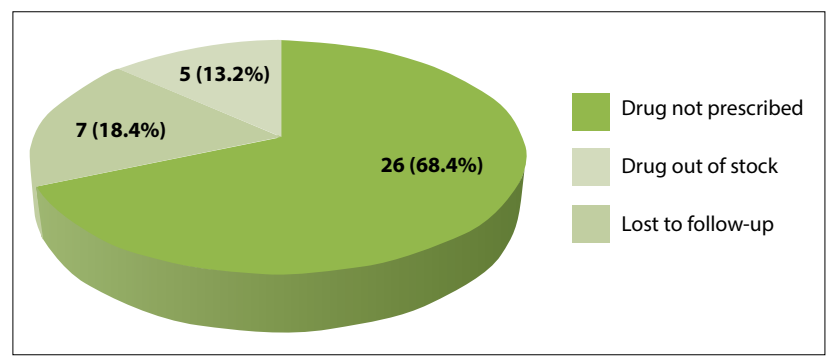

Fig. 1. Reasons for not completing IPT $(\mathrm{N}=38)$. analysis. Of these, $63.5 \%(n=66)$ were female and $36.5 \%(n=38)$ male (Table 1). There was no statistical significance between female and male respondents who completed IPT at 9 months $(p=2.699)$.

Of the subjects, $31.7 \%(n=33)$ completed IPT within 6 months and $63.5 \%(n=66)$ within 9 months. Some could not collect their medication because of the unavailability of isoniazid; however, they did complete IPT within 9 months.

The reasons for $36.5 \%$ of subjects $(n=38)$ not completing the IPT course were identified as unavailability of drugs $(18.4 \%, n=7)$, loss to follow-up $(13.2 \%, n=5)$ and, most importantly, $68.4 \%(n=26)$ to whom IPT was not prescribed (Fig. 1). The clinical records of the affected patients did not state the exact reasons for this.

None of the 66 respondents who completed IPT at the end of 9 months was diagnosed with TB at the conclusion of data gathering. The study findings confirmed the results of other studies that IPT for at least 6 months or up to 9 months was successful in preventing TB. Incidence rates of 0.80 per 100 person-years ${ }^{[9]}$ and 2.3 per 100 person-years ${ }^{[10]}$ have been reported elsewhere.

Only $5(13.2 \%)$ of the subjects who did not complete IPT were responsible for not doing so. The other 33 (86.5\%) did not complete IPT because isoniazid was either not prescribed or out of stock.

\section{Discussion}

It is a cause for concern that IPT was either not prescribed or unavailable in so many cases, since the study results confirm the findings of other studies that IPT for at least 6 months and up to 9 months successfully prevented TB.

To ensure quality healthcare, the required drugs must be available. The current national treatment guidelines in $\mathrm{SA}^{[11]}$ recommend the use of a TST before commencing IPT. This practice should be standardised to ensure that the appropriate duration of IPT can be adhered to in order to implement research evidence, thus promoting evidence-based practice in healthcare delivery. Healthcare workers need to take responsibility for the quality of the healthcare they deliver, including the procurement of drugs to ensure the availability of IPT.

Performance appraisal should include aspects such as timely ordering of medication, accountability for negligence to order medication, and attending workshops and continuing professional development sessions. Healthcare professionals who do not meet their responsibilities in providing quality healthcare as stipulated in their job descriptions should be held accountable for poor quality of healthcare, and the necessary actions should be taken against them.

It was evident that IPT for at least 6 months successfully prevented TB. It therefore remains a serious concern that inadequate healthcare or possible incompetence of healthcare providers were the main reasons for patients not completing IPT despite positive evidence of its effectiveness. It is, however, important to note that the study was conducted in the catchment area of one clinic in one health district, and cannot be generalised to other contexts.

Author contributions. EIO conducted the research, wrote the initial reports and funded the study. LR contributed to writing the proposal,

Table 1. Gender distribution of subjects who completed IPT at 9 months

\begin{tabular}{llll}
\hline Completed at 9 months & Females, $\boldsymbol{n}(\%)$ & Males, $\boldsymbol{n}(\%)$ & Total, $\boldsymbol{n}(\%)$ \\
\hline Yes & $28(42.4)$ & $10(26.3)$ & $38(36.5)$ \\
No & $38(57.6)$ & $28(73.7)$ & $66(63.5)$ \\
Total & $66(100.0)$ & $38(100.0)$ & $104(100.0)$
\end{tabular}


research design and instrument development, data interpretation and writing the report.

1. National Department of Health, South Africa. National Antenatal Sentinel HIV and Syphilis Prevalence Survey in South Africa, 2011. Pretoria: NDoH, 2012.

2. National Department of Health, South Africa. Management of Drug-resistant Tuberculosis: Policy Guidelines. Pretoria: $\mathrm{NDoH}, 2011$.

3. National Department of Health, South Africa. Clinical Guidelines for the Management of HIV \& AIDS in Adults and Adolescents. Pretoria: $\mathrm{NDoH}, 2010$.

World Health Organization. Global Tuberculosis Report 2012. Geneva: WHO, 2012.

5. World Health Organization. Guidelines for Intensified Tuberculosis Case-finding and Isoniazid Preventive Therapy for People Living with HIV in Resource-Constrained Settings. Geneva: WHO, 2011.

6. Granich R, Akolo C, Gunneberg C, Getahun H, Williams P, Williams B. Prevention of tuberculosis in people living with HIV. Clin Infect Dis 2010;50(S3):S215-S222. DOI:10.1086/651494
7. Foundation for Professional Development. The Integrated Management of TB, HIV \& STI in the Primary Healthcare Setting. Pretoria: FPD, 2010.

8. Wilson D, Cotton M, Bekker L, Meyers T, Venter F, Maartens G, eds. Handbook of HIV Medicine. 2nd 8. Wilson D, Cotton M, Bekker L, Meyers T, Venter F,
ed. Cape Town: Oxford University Press, 2008:36.

ed. Cape Town: Oxford University Press, 2008:36.
Golub JE, Pronyk P, Mohapi L, et al. Isoniazid preventive therapy, HAART and tuberculosis risk in Golub JE, Pronyk P, Mohapi L, et al. Isoniazid preventive therapy, HAART and tuberculosis risk in
HIV-infected adults in South Africa: A prospective cohort. AIDS 2009;23(5):631-636. DOI:10.1097/ HIV-infected adults in So

10. Rangaka MX, Wilkinson RJ, Boulle A, et al. Isoniazid plus antiretroviral therapy to preven tuberculosis: A randomised double-blind, placebo-controlled trial. Lancet 2014;384(9944):682-690. DOI:10.1016/S0140-6736(14)60162-8

11. National Department of Health, South Africa. National Consolidated Guidelines for the Prevention of Mother-to-Child Transmission of HIV (PMTCT) and the Management of HIV in Children, Adolescents and Adults. Pretoria: NDoH, 2014.

Accepted 18 March 2016. 